\title{
Effects of Wind Disturbance on the Aerodynamic Performance of a Quadrotor MAV during Hovering
}

\author{
Yao Lei $\mathbb{D}^{1,2}$ Yiyong Huang $\mathbb{D}^{1}{ }^{1}$ and Hengda Wang $\mathbb{D}^{1}$ \\ ${ }^{1}$ School of Mechanical Engineering and Automation, Fuzhou University, Fuzhou 350116, China \\ ${ }^{2}$ Key Laboratory of Fluid Power and Intelligent Electro-Hydraulic Control (Fuzhou University), Fujian Province University, \\ Fuzhou 350116, China \\ Correspondence should be addressed to Yao Lei; yaolei@fzu.edu.cn
}

Received 17 October 2020; Revised 10 March 2021; Accepted 10 April 2021; Published 22 April 2021

Academic Editor: Jesús Lozano

Copyright (c) 2021 Yao Lei et al. This is an open access article distributed under the Creative Commons Attribution License, which permits unrestricted use, distribution, and reproduction in any medium, provided the original work is properly cited.

Wind disturbance could render thrust and power variation or even causing roll which is difficult to maintain a steady flight in gust especially when the horizontal or vertical wind is involved. In this paper, the horizontal wind and vertical wind are presented to study the influence of wind disturbance on aerodynamic characteristics of the quadrotor aircraft in hovering by experiments and numerical simulations. First, the simplified aerodynamic model with the wind disturbance was analyzed in detail. Also, the lowspeed wind tunnel tests were performed to obtain the thrust and power variation of the quadrotor aircraft with rotor spacing ratio $s=1.1-1.8$ in both horizontal and vertical winds of $0-5 \mathrm{~m} / \mathrm{s}$ with the rotational speed ranging from 1500 to $2300 \mathrm{rpm}$. Finally, the simulations are performed by utilizing the Computational Fluid Dynamics (CFD) software ANSYS to study the flow field distribution of quadrotor with the influence of the wind disturbance. The comparison between experimental results and simulation results shows that the quadrotor achieves better aerodynamic performance with larger thrust and smaller power consumption at rotor spacing ratio $s=1.8$. Additionally, the quadrotor can effectively resist the horizontal wind disturbance, which will bring larger power loading for the quadrotor, especially at $2.5 \mathrm{~m} / \mathrm{s}$. However, the vortices near blade-tip move upwards and deform with the influence of vertical wind, resulting in the reduction of thrust and aerodynamic performance of the quadrotor.

\section{Introduction}

Since their development, micro aerial vehicles (MAVs) have been widely used in military and civil fields with their flexible structure and good stability [1-4]. Despite the many advantages of MAVs, there are still many challenges on the design of stability and flight efficiency of MAVs. When the quadrotor aircraft flies in a city terrain, the wind speed generally does not exceed $5 \mathrm{~m} / \mathrm{s}$ at a flight height of $20 \mathrm{~m}$ [5]. At this time, not only there is strong aerodynamic interference between adjacent rotors, but also the effect of the oncoming flow is needed to be considered. Generally, the horizontal and vertical components of wind direction are more significant in contributing to roll disturbances. These are a result of a thrust difference across the span of the aircraft where the horizontal component primarily affects the velocity vector and the vertical component affects the angle of attack [6]. The mutual induction of rotor wakes and the interaction of vortices make the wakes twisted, wound, and broken when the wind disturbance is introduced. Therefore, the downwash flow of MAVs will be disorganized and cause extra power consumption. Eventually, the wind will pose on MAVs and affect the flight efficiency with thrust and power variation. Because of this, a rotor-wing MAV consumes more energy than a fixed-wing MAV with the same mass. However, proper rotor arrangement is beneficial to reduce the power consumption or even improve the flight efficiency of propulsion systems, especially when the wind disturbance is involved in this case.

At present, the studies on MAVs are mainly focused on the trajectory tracking and attitude control. Few of them are involved with power requirements or aerodynamic characteristics. For trajectory tracking and attitude control, Ha et al. [7-10] proposed a trajectory tracking method with collision avoidance for a multicopter based on geometrical 
relations, but only gentle wind $(3.4 \mathrm{~m} / \mathrm{s}-5.4 \mathrm{~m} / \mathrm{s})$ is considered external turbulence in this algorithm. For a desired mission at low altitude through various terrains, stability and control, aerodynamic performance, and endurance offer significant challenges in achieving the quasisteady flight. Thus, for power requirements, Sheng et al. [11-13] developed a steady-state control and optimization scheme with minimum power consumption based on the mathematical model of the electric variable-pitch propeller to give enhanced MAV roll control. In [14], the blade-flapping response of a quadrotor propeller is analyzed in order to identify the aerodynamic moment acting on a propeller in the presence of a wind gust. However, only experiments are involved on an isolated rotor where the wind field produced by a set of fans with fluctuating wind velocity. For aerodynamic characteristics, Mohamed et al. [15] studied aerodynamic characteristics of MAVs in the turbulent wind flow conditions around a building configuration by Computational Fluid Dynamics (CFD) method. The CFD application for the MAVs is limited to the region near the building. Lei and Wang $[16,17]$ analyzed the aerodynamic performance and flow field characteristics of quadrotor-MAV by experiments and numerical simulation. It is founded that the rotors pacing at $L / R=3.6$ ( $L$ is rotor spacing between the center of adjacent rotors, and $R$ is the radius of the rotor) with a larger thrust and smaller power are considered to be the best aerodynamic configuration for the quadrotor in hover. Hwang et al. [18] studied the wake interaction of the quadrotor on the hover and forward flight by numerical simulations. It proved that a proper CFD method is able to predict wind gust of buildings for MAVs in an urban environment. Nguyen et al. $[19,20]$ analyzed the lift coefficient and drag coefficient of quadrotor MAV by wind tunnel tests which proved its ability to attenuate external disturbances such as wind gusts.

According to the above researches, to understand how turbulence influences the aerodynamic performance and energy consumption, it is worth to analyze the thrust and power variation to characterize the flight efficiency relevance to MAVs. In particular, the flow field distribution of the quadrotors and the aerodynamic interference between the rotors is further complicated by the wind disturbance which induces vortex deformation and movement of the rotor wakes which need to be further addressed. The main purpose of this paper is to study the wind effect on thrust and power consumption for quadrotor in hover with different rotor arrangement, which may provide the appropriate recommendations for a future study on control strategies.

The structure of this paper is organized as follows: in Section 2, the aerodynamic model of quadrotor is established in detail. In Section 3, thrust and power consumption are analyzed by a custom-designed experiment platform. In Section 4, CFD simulations are performed to study flow field characteristics of the quadrotor. Finally, Section 5 gives the conclusions.

\section{Theoretical Analysis}

2.1. Aerodynamic Model of Quadrotor. The flow field of quadrotor MAV is shown in Figure 1. The adjacent rotors

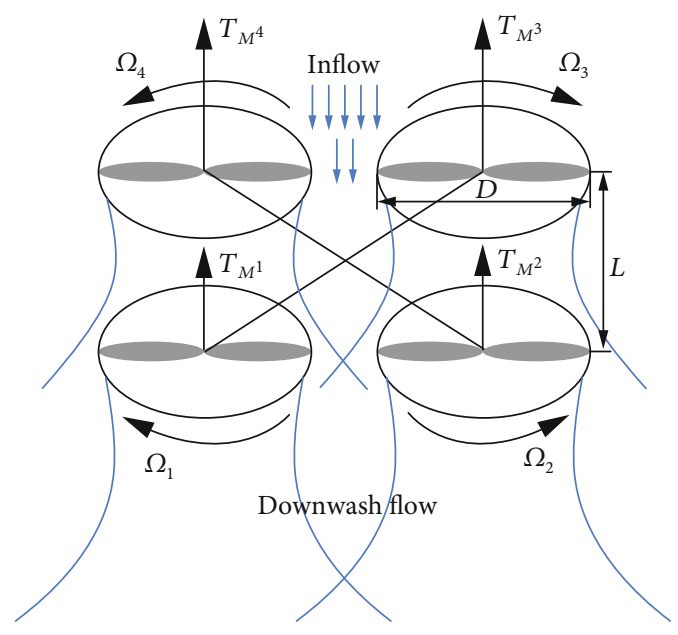

Figure 1: Dynamic model of quadrotor-MAV.

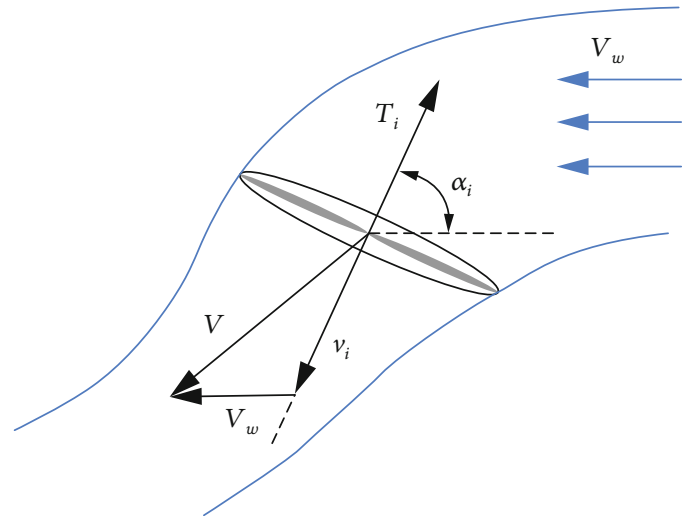

Figure 2: Wind disturbance model of rotors.

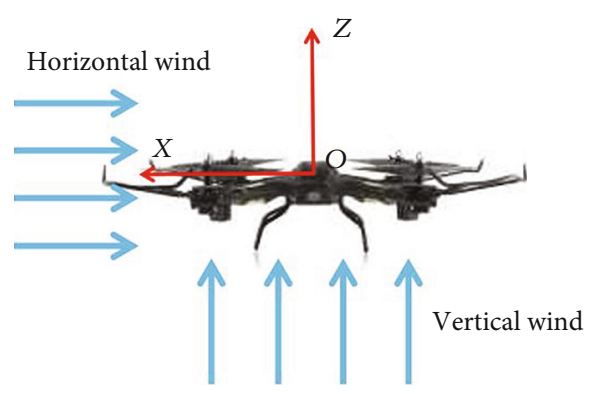

Figure 3: Direction of the wind.

rotate clockwise and counterclockwise, so the torque generated by the rotation of the rotors is offset. A quadrotor aircraft can achieve vertical motion, pitch, roll, yaw, and other attitude by changing the rotational speed. In Figure $1, T, \Omega, b, L$, and $D$ are the thrust, the rotational speed, the proportionality coefficient, the distance between the rotating center of two adjacent rotors, and the diameter of rotor, respectively.

With no wind disturbance, thrust of the quadrotor can be defined as

$$
T=\sum_{i=1}^{4} T_{M_{i}}=\sum_{i=1}^{4} b \Omega_{i}^{2} \quad(b>0, i=1,2,3,4) .
$$




\section{$\bullet$}

(a)

(b)

FIgURE 4: Rotor profile: (a) top view; (b) side view.

TABLE 1: Rotor parameters.

\begin{tabular}{lcccccc}
\hline Radius $(\mathrm{m})$ & Blade pitch $(\mathrm{m})$ & Twist $(\mathrm{deg})$ & $\mathrm{RPM}^{1}(\mathrm{RPM})$ & Reynolds number $\left(10^{5}\right)$ & Max camber $(\%)$ & Mach number $(-)$ \\
\hline 0.2 & 0.157 & 0 & $1500 \sim 2300$ & $0.75 \sim 1.25$ & 5.5 & $0.1 \sim 0.15$ \\
\hline
\end{tabular}

${ }^{1}$ RPM: Revolutions Per Minute.

2.2. Wind Disturbance Model for Rotor. Each rotor of the MAV will gain additional thrust with the influence of wind disturbance. The wind disturbance model of a rotor is shown in Figure 2.

The total thrust $T_{i}$ for each rotor can be expressed as [19]

$$
\begin{aligned}
& T_{i}=2 \rho A V v_{i}, \\
& V=V_{w}+v_{i},
\end{aligned}
$$

where $\rho$ is the air density and $A$ is the area of the rotor disk. $v_{i}$ , $V_{w}$, and $V$ are the induced speed of rotor, wind speed, and the total induced speed, respectively.

Further, the total induced speed $V$ can be transformed to

$$
V=\sqrt{\left(V_{w} \cos \alpha_{i}+\boldsymbol{v}_{i}\right)^{2}+\left(V_{w} \sin \alpha_{i}\right)^{2}}
$$

where $V_{w}$ and $\alpha_{i}\left(0 \leq \alpha_{i} \leq \pi\right)$ are the wind speed and the angle between the rotor axis and the wind direction. Obviously, the wind is vertical when $\alpha_{i}=0$, and the wind is horizontal when $\alpha_{i}=\pi / 2$.

According to the momentum theory, the power is expressed as

$$
P=T_{i}\left(v_{i}+V_{w} \cos \alpha_{i}\right)
$$

Thus, wind from any direction can be divided into horizontal and vertical components. For a MAV immerged in a wind field, the horizontal component affects the velocity vector of the rotor, and the vertical component affects the angle of attack of the aircraft. In this paper, both the horizontal wind and vertical wind are applied to study the effects of wind disturbance on the aerodynamic characteristics of quadrotors. The diagram of wind direction is shown in Figure 3.

Normally, the wind disturbance environment is generally light air $(0.3-1.5 \mathrm{~m} / \mathrm{s})$, light breeze $(1.6-3.3 \mathrm{~m} / \mathrm{s})$, and gentle breeze $(3.4-5.4 \mathrm{~m} / \mathrm{s})$ when the flight height of MAVs is lower than $20 \mathrm{~m} \mathrm{[21].} \mathrm{Thus,} \mathrm{the} \mathrm{wind} \mathrm{speed} \mathrm{studied} \mathrm{in} \mathrm{this} \mathrm{paper}$ does not exceed $5 \mathrm{~m} / \mathrm{s}$.

In order to study the influence of rotor spacing on the aerodynamic performance of quadrotors, the spacing ratio $s$ is defined by the distance $L$ between the rotating center of two adjacent rotors and the diameter of rotor $D$ as follows:

$$
s=\frac{L}{D} .
$$

TABLE 2: Experimental parameters.

\begin{tabular}{lccc}
\hline Wind direction & $\begin{array}{c}\text { Wind speed } \\
(\mathrm{m} / \mathrm{s})\end{array}$ & $\begin{array}{c}\text { Rotor spacing } \\
\text { ratio } s\end{array}$ & $\begin{array}{c}\text { RPM } \\
(\mathrm{rpm})\end{array}$ \\
\hline $\begin{array}{l}\text { Horizontal, } \\
\text { vertical }\end{array}$ & $0,2.5,4.0$ & $1.1 \sim 1.8$ & $1500 \sim 2300$ \\
\hline
\end{tabular}

In this case, the spacing $L$ is ranged from $1 D$ to $2 D(D$ is the diameter of the rotor). The minimum $L=1.1 \mathrm{D}$ is set to avoid the collision of adjacent rotors, and the maximum $L$ $=1.8 \mathrm{D}$ is set to avoid oversize of the MAV since a larger rotor spacing $(L>1.8 D)$ will lead to a long rotor arm with extra weight which is proved not beneficial to improving maneuverability with decreased performance.

For MAVs, a Figure of Merit (FM) often is promoted to characterize the rotor efficiency in hover. In this paper, FM can be defined as follows [21]:

$$
\begin{gathered}
\mathrm{FM}=\frac{C_{T}^{3 / 2}}{\sqrt{2} C_{P}}=\frac{T^{3 / 2}}{P \sqrt{2 \rho A}}=\frac{T^{3 / 2}}{Q \Omega \sqrt{2 \rho A}}, \\
C_{T}=\frac{T}{\rho A \Omega^{2} R^{2}}, \\
C_{P}=\frac{P}{\rho A \Omega^{3} R^{3}}=\frac{Q}{\rho A \Omega^{2} R^{3}},
\end{gathered}
$$

where $R$ is the radius of the rotor and $C_{T}, C_{P}$, and $Q$ are the thrust coefficient, the power coefficient, and the torque, respectively.

Also, power loading (PL) can be used to evaluate the aerodynamic performance of quadrotor, which is given as follows:

$$
\mathrm{PL}=\frac{C_{T}}{\Omega R C_{P}}=\frac{T}{\mathrm{Q} \Omega}
$$

Both FM and PL are proposed as the metric of the rotor system which is related to the thrust produced by the power required. When designing a vehicle, the aim was to maximize the flight efficiency such that energy requirements are minimized. This will give the vehicle the best endurance or payload capabilities possible. 


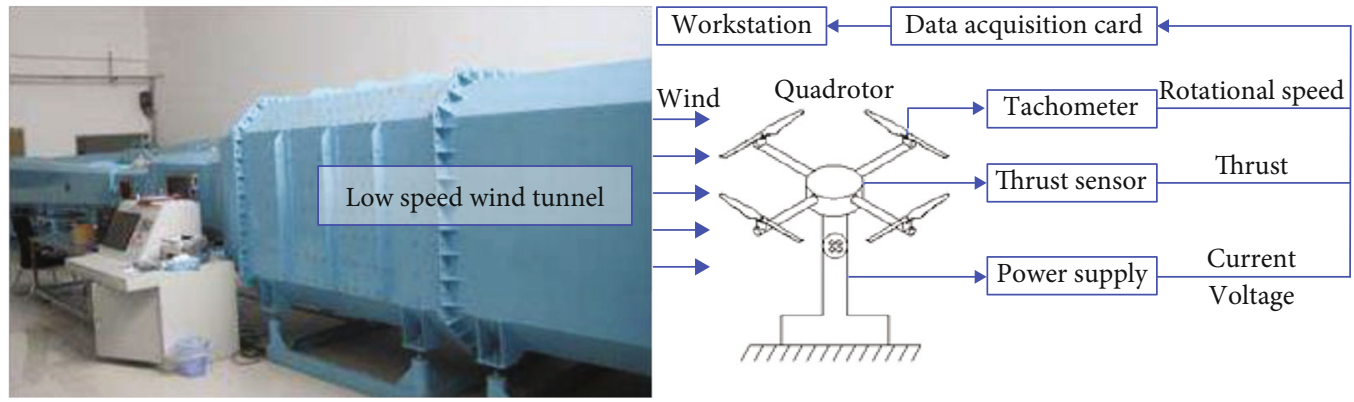

FIGURE 5: Sketch of experimental setup.

\section{Wind Tunnel Tests}

3.1. Basic Parameters. In this paper, the rotor is manufactured by carbon fiber with a diameter of $400 \mathrm{~mm}$. The rotor has two blades with the total mass of $0.015 \mathrm{~kg}$. The rotor profile is presented in Figure 4, and the rotor parameters are shown in Table 1 in detail. Specially, to explore the wind effect of rotor spacing on the aerodynamic performance of quadrotor in hover, the rotor speed is set at $2200 \mathrm{rpm}$ as its work mode. In this case, $2200 \mathrm{rpm}$, as an appropriate speed consuming reasonable power to meet the basic requirements, was chosen in this manuscript to discover the optimal rotor spacing.

3.2. Experimental Setup. Considering that the normal wind speed in the natural environment generally does not exceed $5 \mathrm{~m} / \mathrm{s}$, light breeze $(1.6 \sim 3.3 \mathrm{~m} / \mathrm{s})$ and gentle breeze $(3.4 \sim 5.4 \mathrm{~m} / \mathrm{s})$ are chosen to conduct the wind tunnel test with horizontal wind and vertical wind for a quadrotor. A lowspeed wind tunnel with an open-jet test section ( 3 m length $\times 3$ m width $\times 2.5 \mathrm{~m}$ height) and honey comb is applied to simulate the natural wind. A settling chamber is attached before the test section to characterize the output wind. Wind is generated with two $3 \mathrm{~m}$ diameter, $45 \mathrm{~kW}$ fans. A top speed of $50 \mathrm{~m} / \mathrm{s}$ could be achieved within the test section. However, for the current low Reynolds number experiments, the maximum testing velocity was only $12.5 \mathrm{~m} / \mathrm{s}$. The extended wind tunnel's flow characteristics are analyzed by an anemometer for velocity distribution from the opening. The local velocity differences in the approach flow at the nozzle exit area were less than $1.3 \%$. The MAV mounted on the test rig which can rotate a certain angle. Because the wind tunnels only produced the horizontal wind, the object was rotated by $90^{\circ}$ in the wind tunnel relative to the airflow when the effect of vertical wind on the quadrotor was studied. As a comparison, the aerodynamic performance of the quadrotor without wind disturbance was also tested. The experimental parameters are presented in Table 2, and the sketch of experimental setup is shown in Figure 5.

As shown in Figure 5, rotors of quadrotors were driven by DC brushless motors (model: EM2835), and the rotational speed of motors can be controlled by pulse width modulation (PWM) from a remote controller. The tachometer (model: DT-2234C, accuracy: $6 \pm(0.05 \%+1 \mathrm{~d}))$ was used to measure the rotational speed of rotors. The thrust of rotor was measured by thrust sensor (model: CZL605, accuracy:

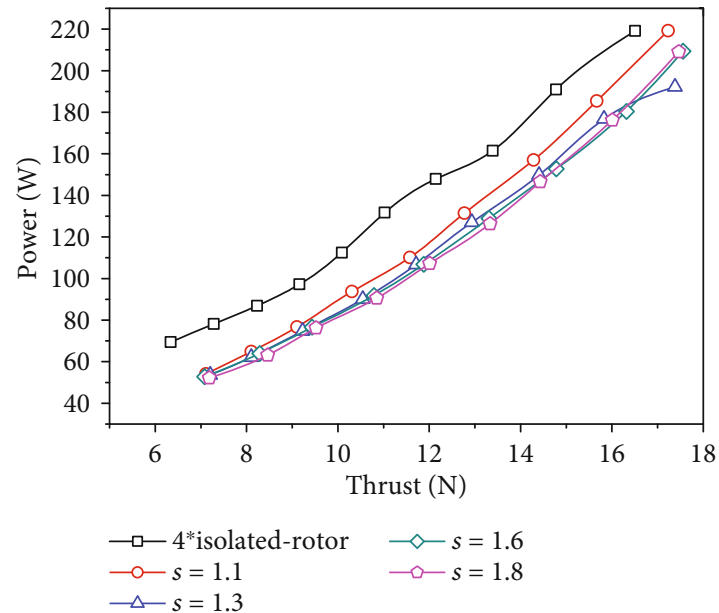

FIgURE 6: Thrust and power variation without wind disturbance.

$0.02 \%$ FS). Power consumption was calculated by current and voltage. Finally, all data was processed by the workstation through the data acquisition card.

The main sources of error in the experiments are the standard deviations of the rotational speed and the mean voltages from the thrust sensors. Typical values of the standard deviations of thrust are about $1 \%$ of the mean values. The values of uncertainty presented in this study are all calculated for 95\% confidence levels. Finally, according to the Kline-McClintock method [17], typical uncertainties in the nondimensional performance coefficients, $C_{T}$, and $C_{P}$ are $1.6 \%, 1.5 \%$, and $1.5 \%$ for $\mathrm{FM}$.

3.3. Experimental Results. Without wind disturbance, an isolated rotor is assumed not subject to any aerodynamic interference, which can be compared with the quadrotor with rotor interference to qualify the variation of the rotor interference, specifically thrust variation and power consumption in this paper. Figure 6 shows the variation of thrust with power consumption of quadrotors with typical rotor spacing compared with isolated rotor when there is no wind disturbance.

According to Figure 6, it is clear that the quadrotor obtained more thrust than four isolated rotors without rotor interference. This indicates that the moderate aerodynamic interference between adjacent rotors is conducive to increase the thrust of the quadrotors. Additionally, there is extra 

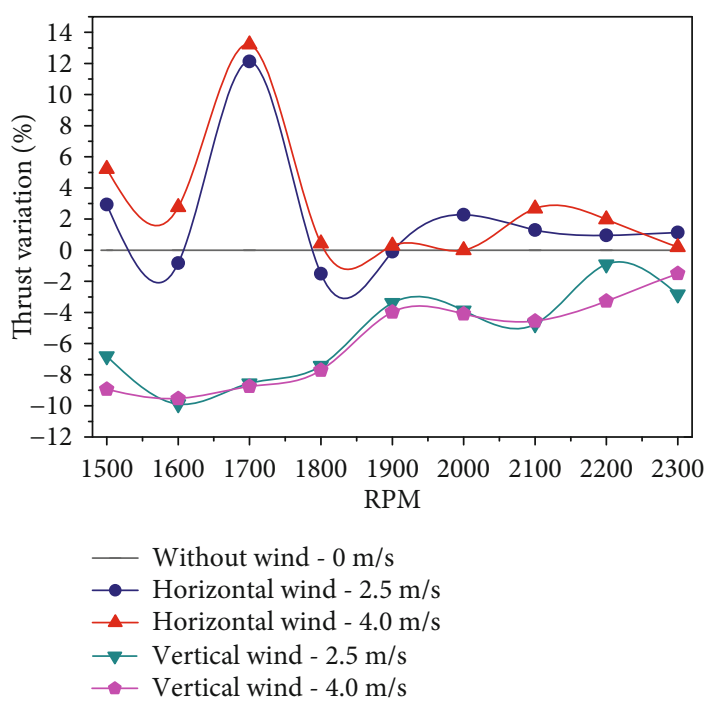

FIGURE 7: Thrust variation with the horizontal and vertical wind.

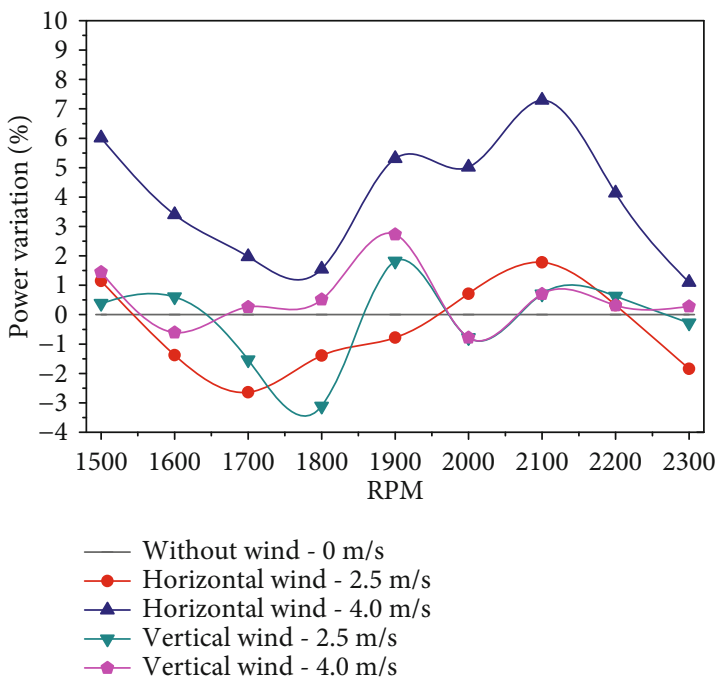

FIGURE 8: Power variation with the horizontal and vertical wind.

power consumption at $\boldsymbol{s}=\mathbf{1 . 1}$, which is characterized by strong rotor interference caused by vibrations. With the increase of rotor spacing, the quadrotors gain more thrust and less power consumption due to reduced interference between adjacent rotors. Especially, it can be seen that quadrotors have better aerodynamic performance with the thrust increment of $5.3 \%$ and the power consumption reduction of $4.6 \%$ at spacing ratio at $\boldsymbol{s}=\mathbf{1 . 8}$ compared with that at $\boldsymbol{s}=\mathbf{1 . 1}$. It is interesting to note that moderate interference may improve the performance without extra power consumption.

Figure 7 shows the thrust variation of quadrotor with the influence of horizontal and vertical wind at $\boldsymbol{s}=\mathbf{1 . 8}$.

As shown in Figure 7, the thrust of the quadrotors increases obviously with the influence of horizontal wind, and it increases further as the wind speed increases. Especially, the maximum increment is reached $13.21 \%$ at 1700 RPM when the horizontal wind speed is $4.0 \mathrm{~m} / \mathrm{s}$. In this case, the rotor interference may offset wind effect to some extent.

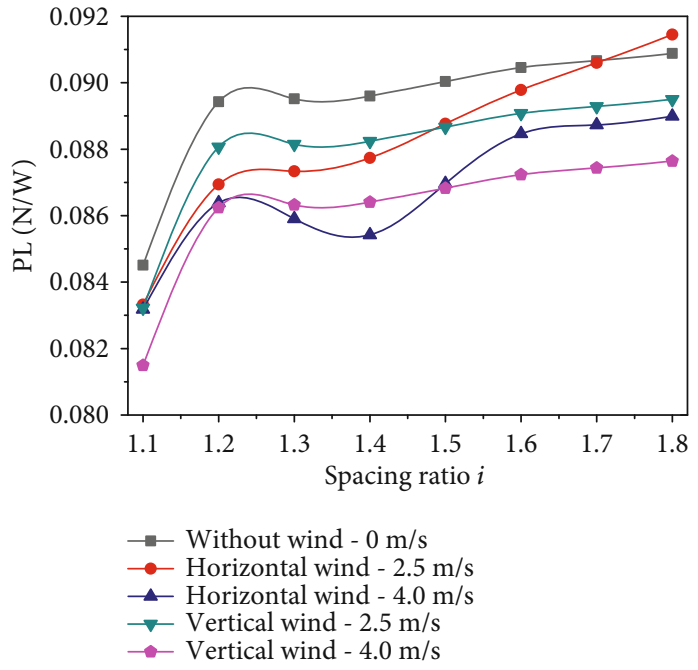

FIGURE 9: PL variation with the horizontal and vertical wind.

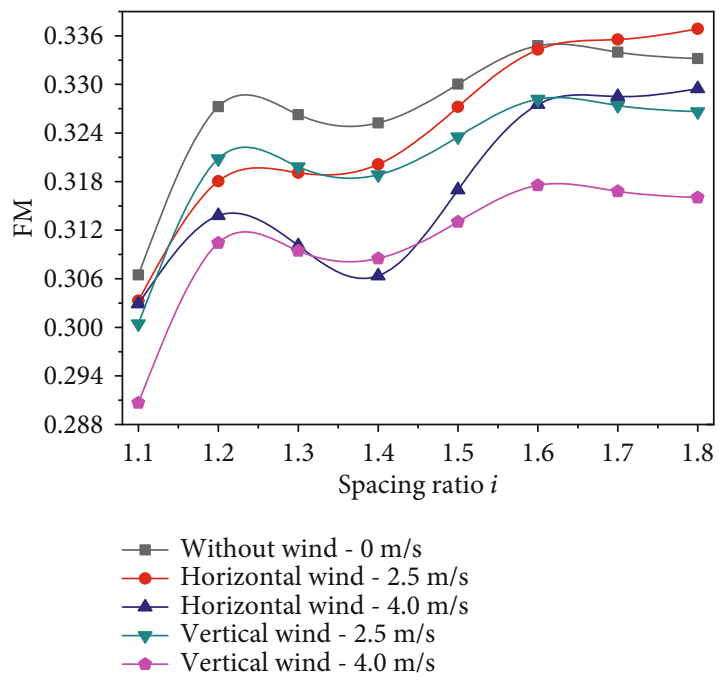

Figure 10: FM variation with the horizontal and vertical wind.

However, the thrust of the quadrotors is reduced with the vertical wind disturbance, especially for a lower RPM (1500 RPM-1800 RPM). Compared with the thrust at $0 \mathrm{~m} / \mathrm{s}$, the maximum reduction in thrust is about $9.89 \%$ and $9.54 \%$ for the vertical wind at $2.5 \mathrm{~m} / \mathrm{s}$ and $4.0 \mathrm{~m} / \mathrm{s}$. It indicates that the horizontal wind is inclined to be advantageous to increase the thrust of quadrotors while the vertical wind decreased the performance where the disordered downwash is diverted along with the vertical wind. Thus, only when a quadrotor has larger thrust or smaller power consumption, it can achieve a good aerodynamic performance since it gives higher flight time and efficiency according to equations (7) and (10). Figure 8 shows the power variation with the effects of horizontal and vertical wind at $s=1.8$.

From Figure 8, it can be noted that the power consumption increased with the influence of the horizontal and vertical wind especially at $4.0 \mathrm{~m} / \mathrm{s}$. This means that the quadrotor will consume extra power to maintain a stable state due to increased wind speed. For the horizontal wind speed at 
TABLE 3: CFD setup.

\begin{tabular}{lcccr}
\hline Turbulence model & Solver & Pressure correction & Pressure velocity coupling & Transient formulation \\
\hline Spalart-Allmaras & Transient flow & SIMPLE & PISO & Second order implicit \\
\hline
\end{tabular}
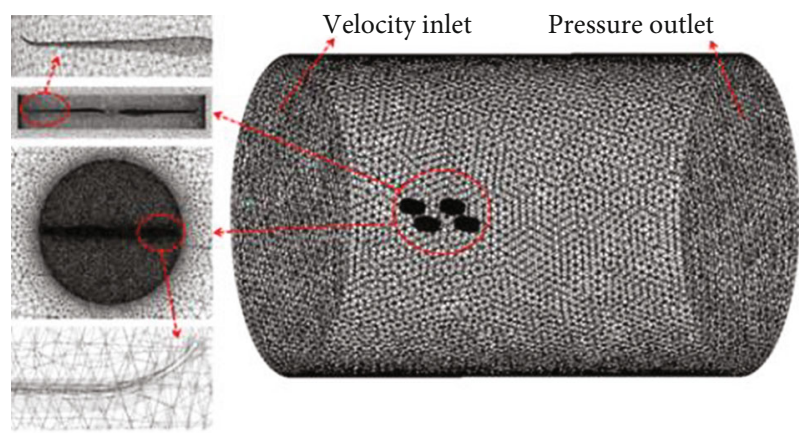

(a)
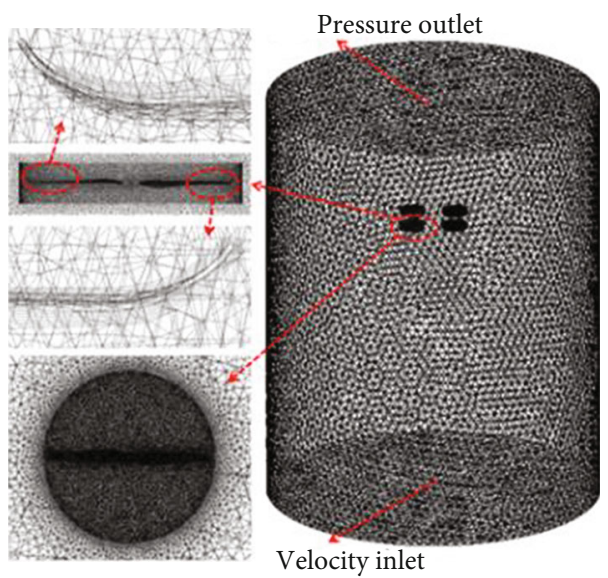

(b)

Figure 11: Mesh distribution: (a) horizontal wind; (b) vertical wind.

$4.0 \mathrm{~m} / \mathrm{s}$, the power increment increases significantly, up to $7.29 \%$ at $2100 \mathrm{rpm}$. Additionally, the power consumption was obviously reduced at $2.5 \mathrm{~m} / \mathrm{s}$ with a lower rotational speed ranged from $1500 \mathrm{rpm}$ to $1800 \mathrm{rpm}$, which indicates that the power consumption can be effectively reduced when the quadrotor is kept at a lower rotational speed with the influence of wind disturbance. The maximum reduction of power consumption is about $2.64 \%$ (horizontal wind $-2.5 \mathrm{~m} / \mathrm{s}$ ) and $3.11 \%$ (vertical wind $-2.5 \mathrm{~m} / \mathrm{s}$ ). Combined with the results in Figure 7, we can conclude that the quadrotor achieved better aerodynamic performance with larger thrust and smaller power consumption with the influence of horizontal wind at $2.5 \mathrm{~m} / \mathrm{s}$.

The power loading of the quadrotor with different rotor spacings and wind speeds at working speed $2200 \mathrm{rpm}$ is shown in Figure 9.

As shown in Figure 9, it can be found that the power loading of the quadrotor is minimum at $s=1.1$ and increases significantly from 1.1 to 1.2 . This is because the strong aerodynamic interference between adjacent rotors at small spacing consumed more power, resulting in a decrease in the performance of the quadrotors. At the same time, more power consumption in wind field decreased the PL compared with no wind disturbance. In addition, the power loading decreases further with the wind speed. Also, when the rotor spacing ratio is larger than $s=1.5(s=1.5-1.8)$, the smaller the wind speed, the larger the power loading. Especially, the quadrotor has the maximum power loading at horizontal wind of $2.5 \mathrm{~m} / \mathrm{s}$ when $s=1.8$. This proves that the quadrotor obtained the best aerodynamic performance when the spacing ratio $s=1.8$, which makes the quadrotor gain more thrust while consuming less power.

Figure 10 shows FM of the quadrotors with different rotor spacings and wind speeds at working speed of 2200 RPM.

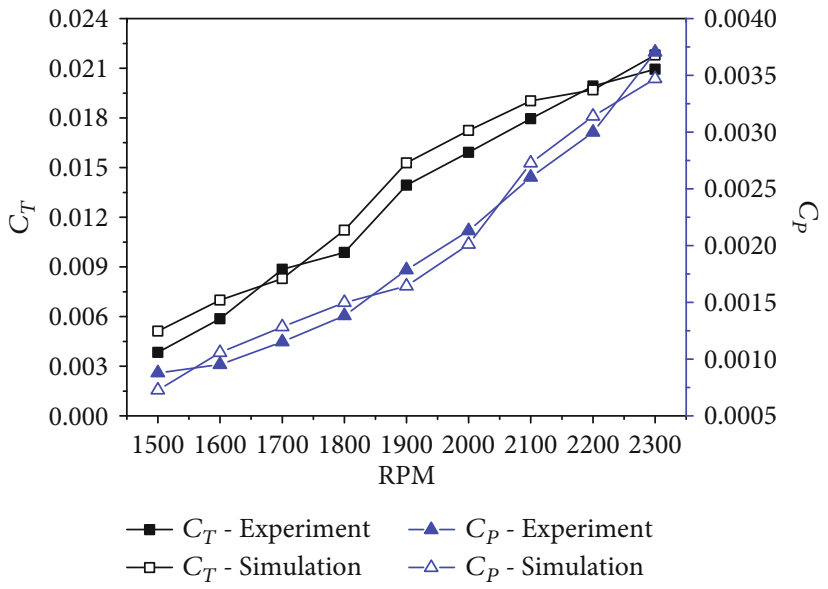

FIGURE 12: Comparison of experiment and simulation.

In Figure 10, a larger FM is observed with spacing ratio $s$ ranging from 1.6 to 1.8 , which shows that the part of the rotor interference offset the wind effect and obtained better hover efficiency. Especially, FM reaches a maximum of 0.336 with horizontal wind of $2.5 \mathrm{~m} / \mathrm{s}$ at $s=1.8$, which indicates that the quadrotors have the best hover efficiency. In addition, FM of the quadrotor is minimum at the small rotor spacing $s=1.1$ which is the same as the PL variation characterized with the strong aerodynamic interference between adjacent rotors.

\section{Numerical Simulations}

4.1. CFD Setup. In this paper, ANSYS is applied to simulate the flow field distribution of a quadrotor system in hover and wind disturbance, and the simulation results are compared with the experimental results to show the 


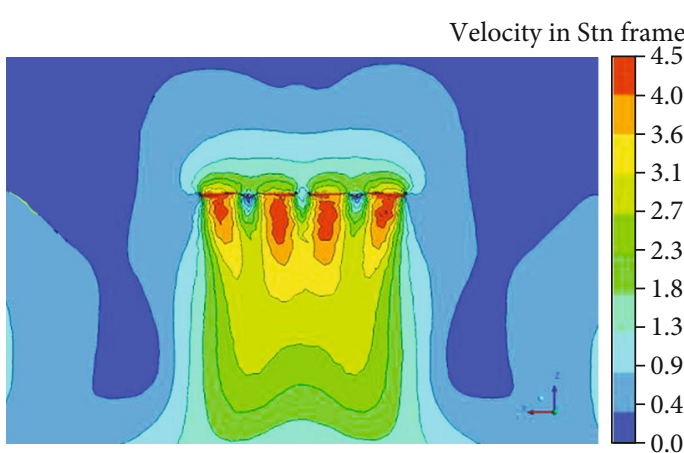

(a)

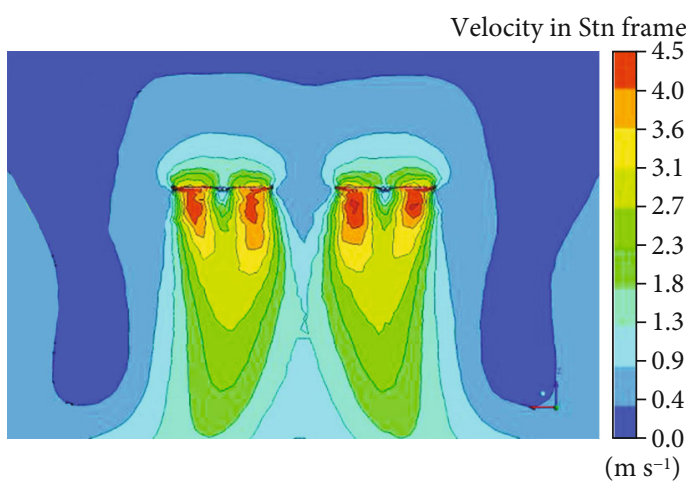

(c)

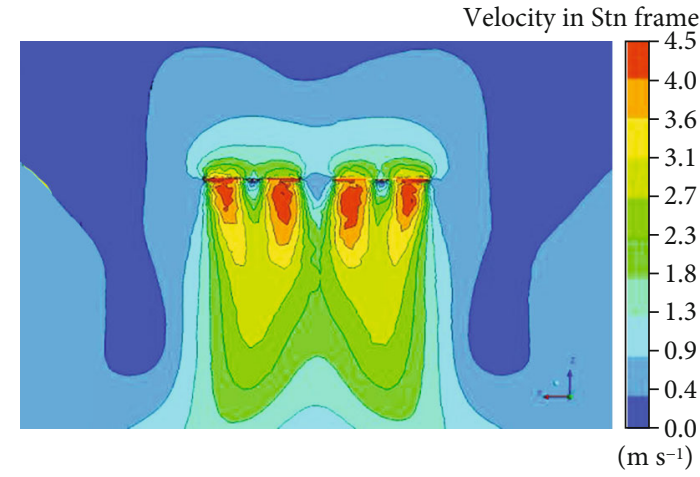

(b)

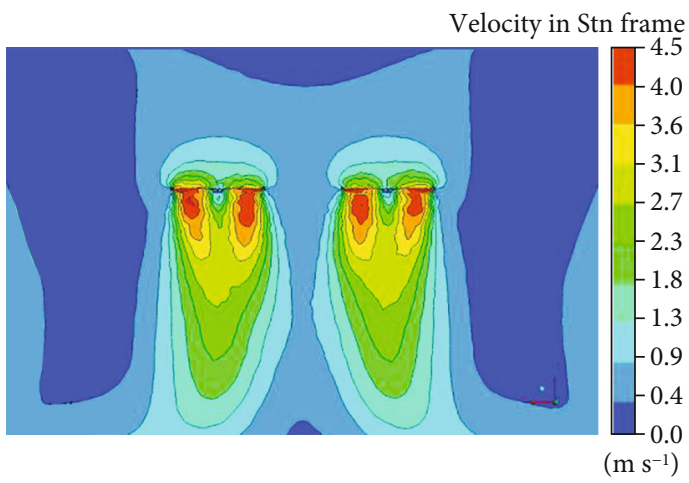

(d)

FIGURE 13: Velocity distribution without wind disturbance with typical rotor spacing: (a) $s=1.1$; (b) $s=1.3$; (c) $s=1.6$; (d) $s=1.8$.

aerodynamic performance of the quadrotors with different rotor arrangement and wind conditions. Table 3 shows the CFD setup.

In this paper, sliding-mesh technique is applied to solve for the motion of the rotors due to the highly unsteady nature of flow in this experiment. For transient flow solver, the adjustable time step based on the dimensionless CourantFriedrichs-Lewy condition (CFL) number of less than one is used for all simulations. The time step size is $10 e-5 \mathrm{~s}$ with 150000 time steps. The mesh distribution is shown in Figure 11.

The computational domain is divided into a cylinder stationary region and four cylinder rotating regions, which has a total size of about 20 million cells. To handle the low Reynolds number flows, multiple viscous boundary layers are used in the simulations, and the max element metrics is below 0.8 to capture the flow detail of the rotor tip and the interfaces between stationary and rotating regions.

In the grid independency study, a formal grid independence study was conducted to show that the results are already reaching the grid independence state. The mesh independence study is shown as follows: (1) run the initial simulation on the initial mesh and ensure convergence of residual error to $10^{-4}$; monitor points are steady and imbalanced below $1 \%$. (2) Once we have met the convergence criteria above for our first simulation, the mesh was refined globally so that we have finer cells throughout the domain. Generally, we would aim for around 1.5 times the initial mesh size. Run the simulation and ensure that the residual error drops below
10-4, that the monitor points are steady, and that the imbalances are below $1 \%$.

In addition, the comparison of experimental results and simulation results is shown in Figure 12. It can be found that the comparison of thrust coefficient $C_{T}$ and power coefficient $C_{P}$ in experiment and simulation showed that they are generally in good agreement, which verifies the effectiveness of simulation.

4.2. Numerical Simulation Results. Velocity distribution of quadrotor without wind disturbance with typical rotor spacing at $2200 \mathrm{rpm}$ is shown in Figure 13.

As shown in Figure 13, it can be found that there is a strong mutual induction between the downwash flows of the adjacent rotors at $s=1.1$, which may increase power consumption by combining the experimental result in Figure 6. With the increase of rotor spacing, the aerodynamic interference between the adjacent rotors gradually decreases. Especially, this aerodynamic interference almost disappears when the rotor spacing ratio is $s=1.8$. The weak aerodynamic interference between adjacent rotors at $s=1.8$ may bring larger thrust or smaller power consumption.

Compared with no wind gusts, the aerodynamic interference between rotors will become more complicated and stronger with the influence of wind disturbance. The velocity distribution between adjacent rotors of quadrotor with wind disturbance at rotor spacing ratio at $s=1.8$ is shown in Figure 14. 


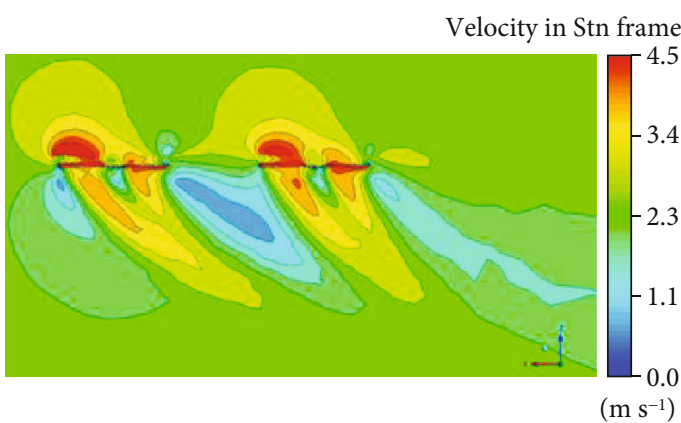

(a)

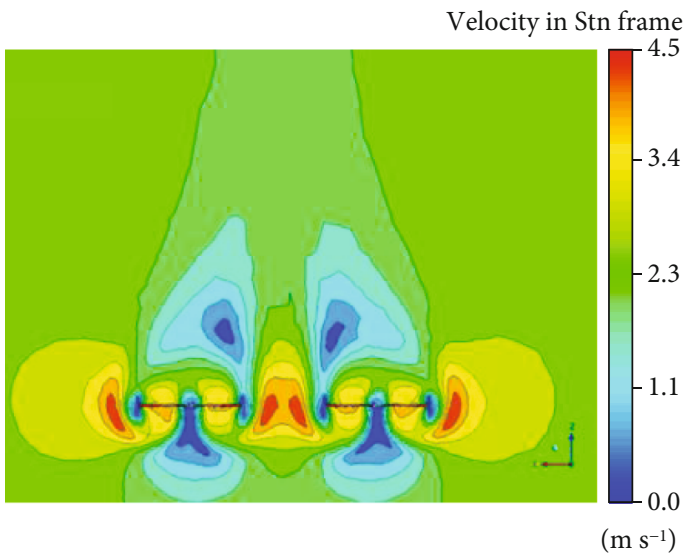

(c)

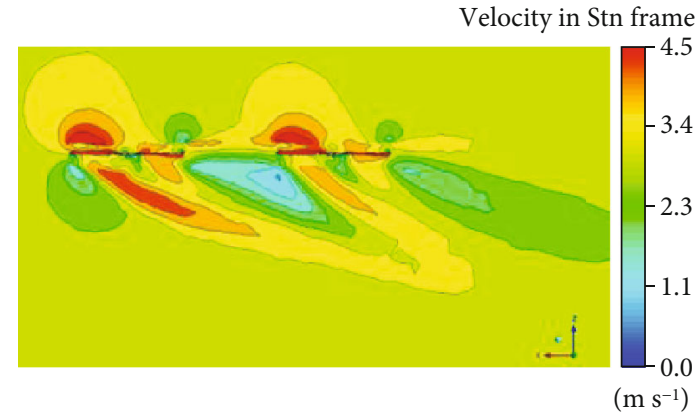

(b)

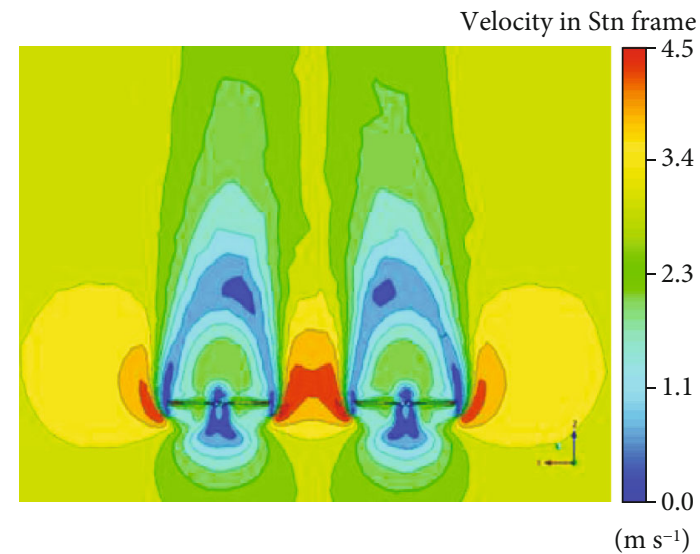

(d)

FIGURE 14: Velocity distribution with wind disturbance: (a)

(a) horizontal wind $-2.5 \mathrm{~m} / \mathrm{s}$;

(b) horizontal wind $-4.0 \mathrm{~m} / \mathrm{s}$;

(c) vertical wind $-2.5 \mathrm{~m} / \mathrm{s}$; (d) vertical wind $-4.0 \mathrm{~m} / \mathrm{s}$.

The velocity distribution affected by horizontal wind is shown in Figures 14(a) and 14(b). Compared with no wind gust, it is clear that the downwash flows of rotors move with the wind and the interaction between the rotors is strengthened with the horizontal wind. This is more obvious at higher wind speed, which may bring larger thrust for rotors. As shown in Figures 14(c) and 14(d), when the vertical wind is introduced, it can be seen that the original downwash flows are blown away compared with no wind gust, which showed a strong negative effect on the aerodynamic performance of the quadrotor without complete downwash shape. Combined with the experimental result in Figures 7 and 8, the negative effect will significantly reduce the thrust of the quadrotors and increases power consumption at the same time, especially for the vertical wind.

The velocity of downwash flow in different wind conditions is shown in Figure 15. When there is without wind disturbance, the velocity of downwash flow velocity gradually decreases with the increase of the distance from the rotor. With the effects of wind disturbance, the velocity of downwash flow fluctuates to a certain extent and finally tends to be stable. Additionally, when $Z / R$ is less than 1.5 , the quadrotor in the horizontal wind obtained a higher downwash velocity than that of the vertical wind which may increase the stability of the quadrotor to resist the wind disturbance ( $Z$ is the $z$-axis which is vertical to the rotational plane of the rotor, and $R$ is the radius of the rotor). This may explain why the thrust of quadrotors with horizontal wind is obviously

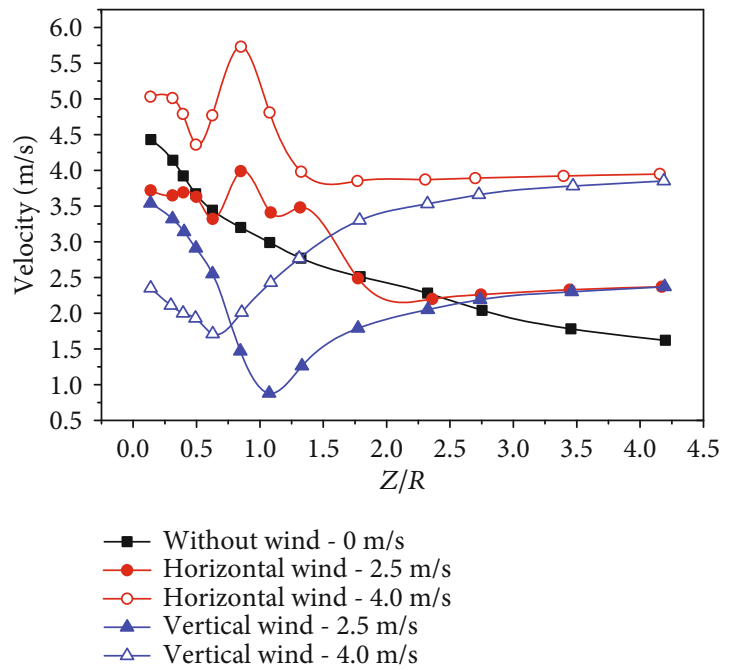

FIgURE 15: Velocity distribution of downwash flow below the quadrotor.

increased, while it decreased with vertical wind. As the wind speed continues to increase, this phenomenon becomes more obvious. Especially, the downwash velocity with the influence of horizontal wind reaches its maximum at about $Z / R=1.0$. Also, the downwash velocity reaches its minimum at about $Z / R=1.0$ because of the influence of vertical wind, which 


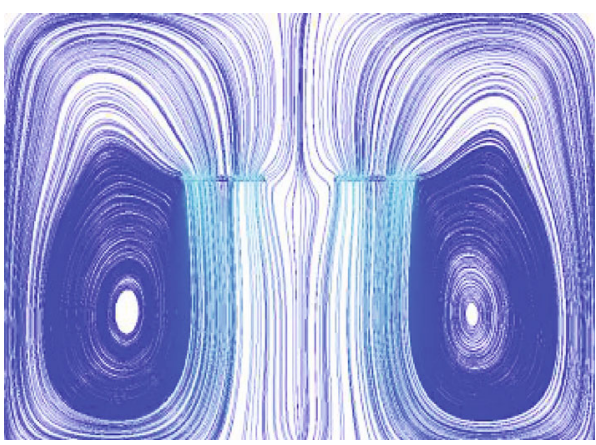

(a)

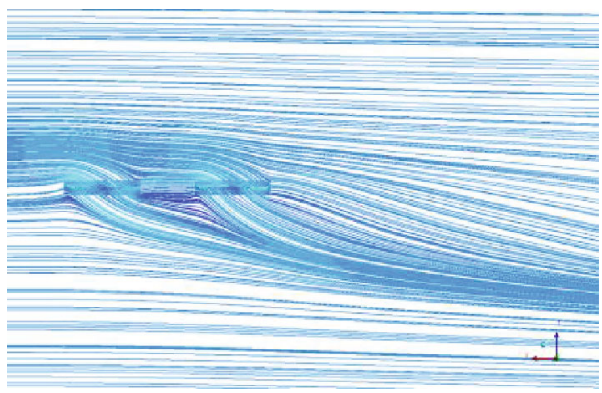

(c)

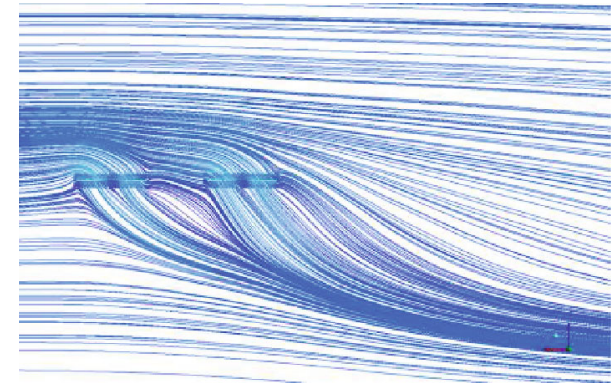

(b)

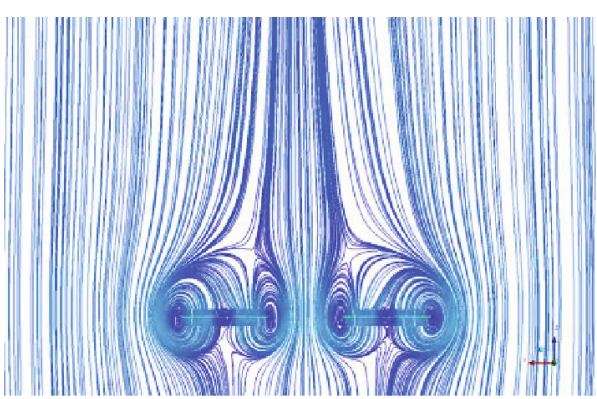

(d)

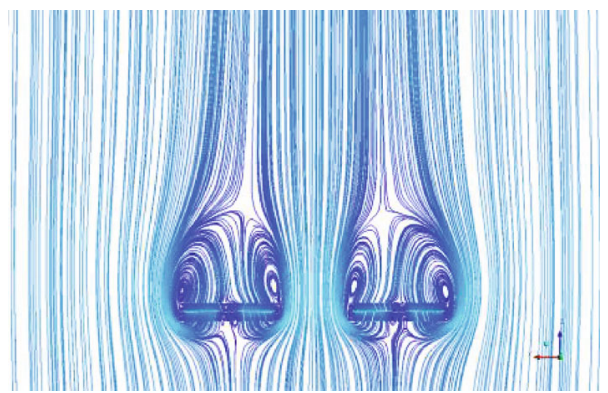

(e)

FIGURE 16: Streamline distribution: (a) without wind disturbance; (b) horizontal wind $-2.5 \mathrm{~m} / \mathrm{s}$; (c) horizontal wind-4.0 m/s; (d) vertical wind $-2.5 \mathrm{~m} / \mathrm{s}$; (e) vertical wind $-4.0 \mathrm{~m} / \mathrm{s}$.

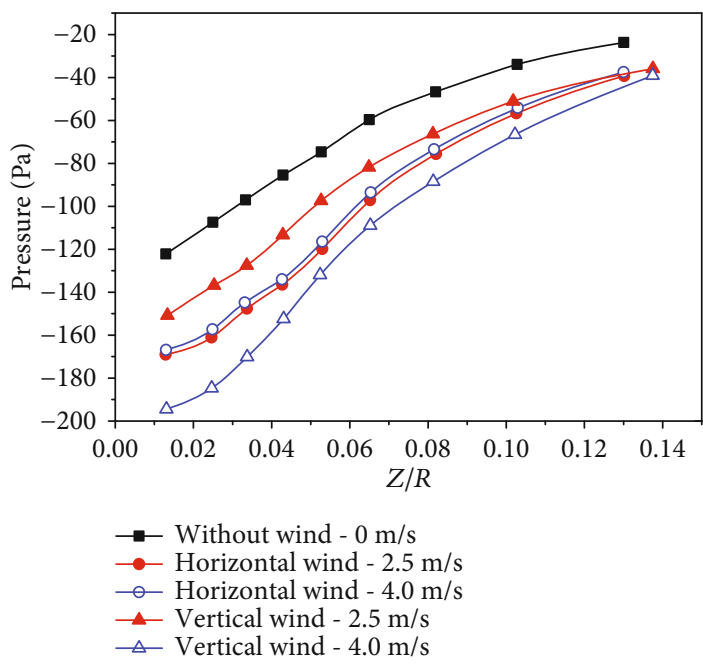

(a)

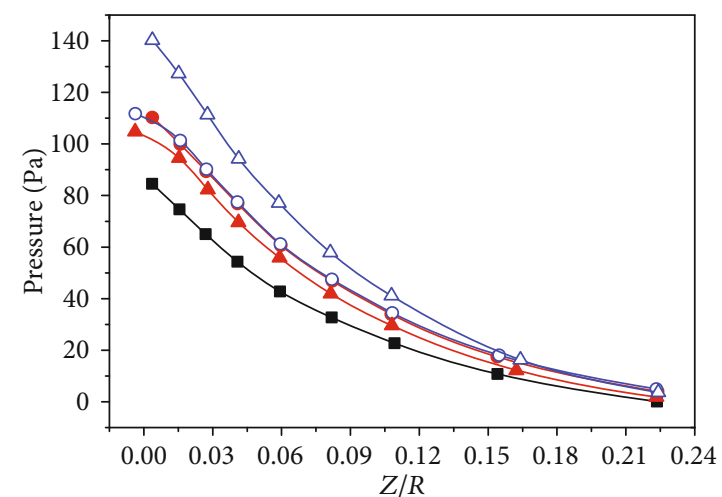

$\rightarrow$ Without wind $-0 \mathrm{~m} / \mathrm{s}$
$\rightarrow-$ Horizontal wind $-2.5 \mathrm{~m} / \mathrm{s}$
$\multimap-$ Horizontal wind $-4.0 \mathrm{~m} / \mathrm{s}$
$\triangle$ Vertical wind $-2.5 \mathrm{~m} / \mathrm{s}$
$\square$ Vertical wind $-4.0 \mathrm{~m} / \mathrm{s}$

(b)

FIGURE 17: Longitudinal pressure distribution of blade tip: (a) above the rotor; (b) below the rotor. 


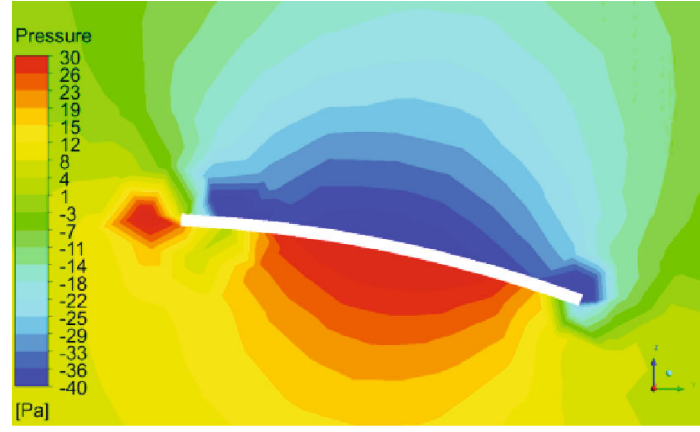

(a)

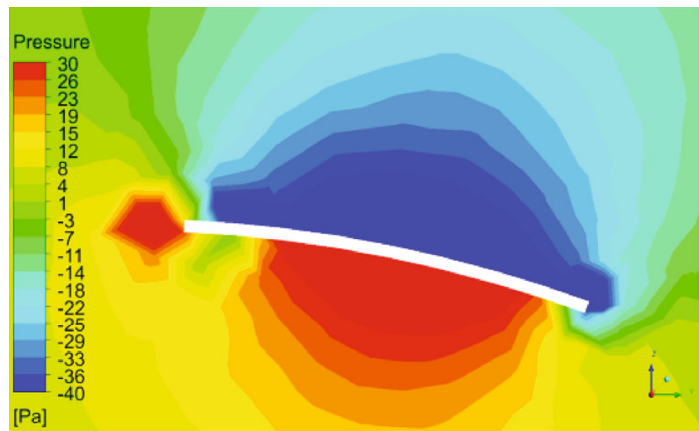

(c)

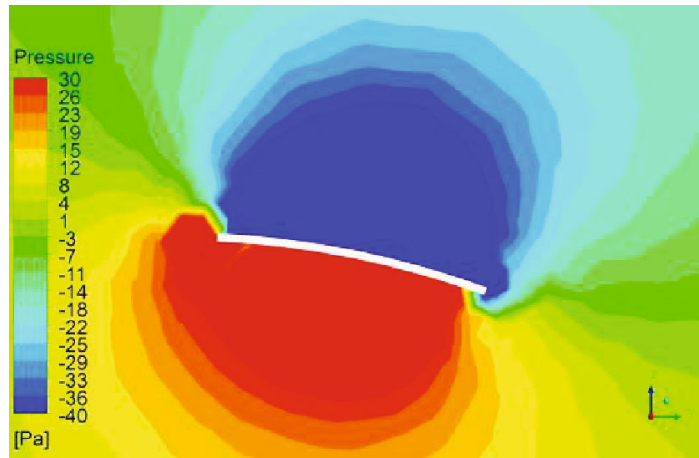

(e)

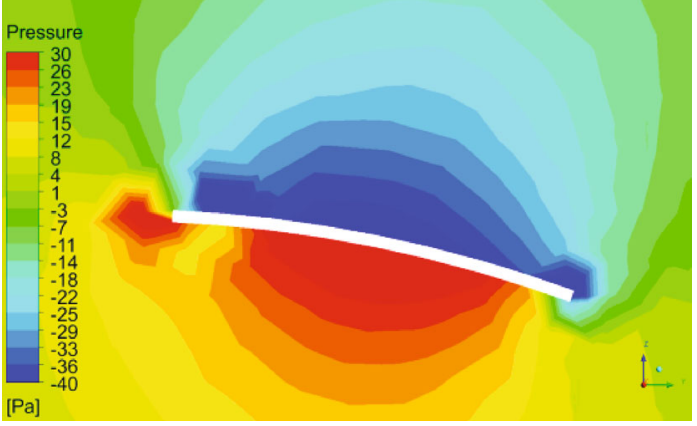

(b)

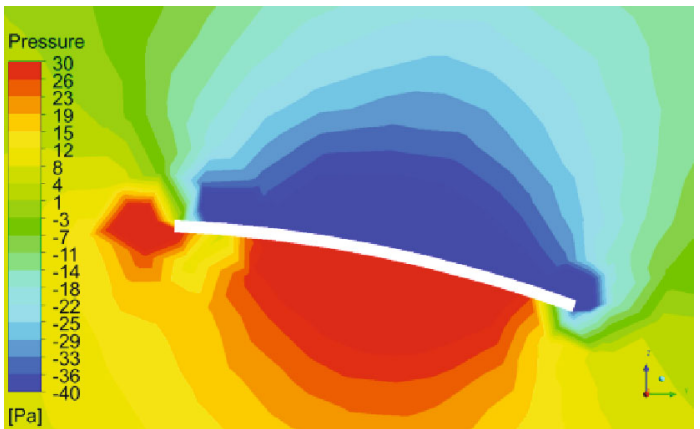

(d)

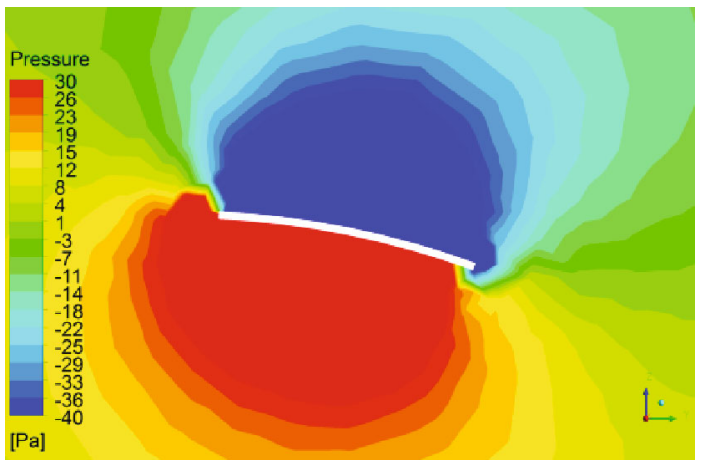

(f)

FIGURE 18: Pressure contour of blade tip with horizontal wind disturbance: (a) $2.5 \mathrm{~m} / \mathrm{s}-1500$ RPM; (b) $4.0 \mathrm{~m} / \mathrm{s}-1500$ RPM; (c) $2.5 \mathrm{~m} / \mathrm{s}-1700 \mathrm{RPM}$; (d) $4.0 \mathrm{~m} / \mathrm{s}-1700 \mathrm{RPM}$; (e) $2.5 \mathrm{~m} / \mathrm{s}-2100 \mathrm{RPM}$; (f) $4.0 \mathrm{~m} / \mathrm{s}-2100 \mathrm{RPM}$.

again indicates that vertical wind will seriously weaken the aerodynamic performance of the quadrotor.

The streamline distribution of the quadrotor with wind disturbance is shown in Figure 16.

From Figure 16(a), it can be found that the streamline distribution is asymmetric with regular vortices and formed below the blade tip when there is no wind disturbance. As shown in Figures 16(b) and 16(c), it is clear that the vortices below the blade tip have disappeared with the influence of horizontal wind. The airflow bypasses the rotor surface to become downwash flow when the horizontal wind acts directly on the rotor disk. It causes the downwash flow to deflect with the wind and mix finally with the horizontal wind. Combined experimental results, it can be inferred why the horizontal wind can bring greater thrust and smaller power for the quadrotor. In addition, with the increase of horizontal wind speed, the streamline distribution becomes clearer and the mutual interference between the downwash flows becomes more obvious. As shown in Figures 16(d) and 16(e), the vortices with the influence of the vertical wind moved upwards and deformed which may cause an increase in power consumption and a decrease in thrust for the quadrotor. The streamline also changes from circumferential flow to axial flow. Compared to the vertical wind of $2.5 \mathrm{~m} / \mathrm{s}$, the vortices continue to move upwards and the extrusion and deformation of the vortices gets worse at the vertical wind of $4.0 \mathrm{~m} / \mathrm{s}$. These may be the reason that the power loading and Figure of Merit of the quadrotor are smaller with the influence of vertical wind compared with that of no wind disturbance and horizontal wind disturbance.

The longitudinal pressure distribution of blade tip with different wind conditions is shown in Figure 17.

As shown in Figure 17, the pressure in the flow field gradually increases along with the surface of the rotor. Additionally, the rotor gains more pressure difference with the influence of horizontal wind, which may increase the thrust 


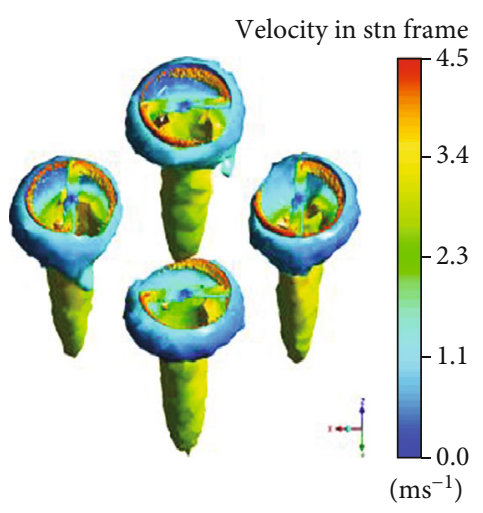

(a)

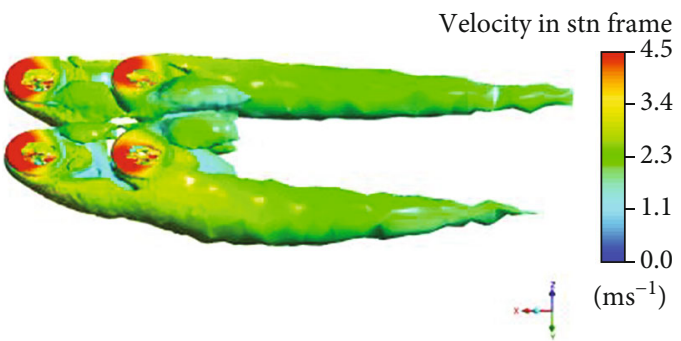

(b)

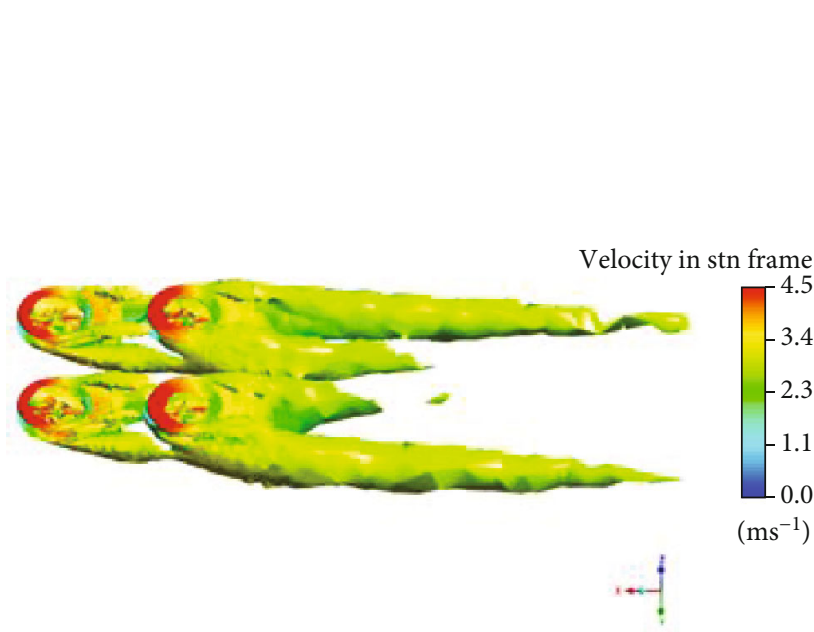

(c)

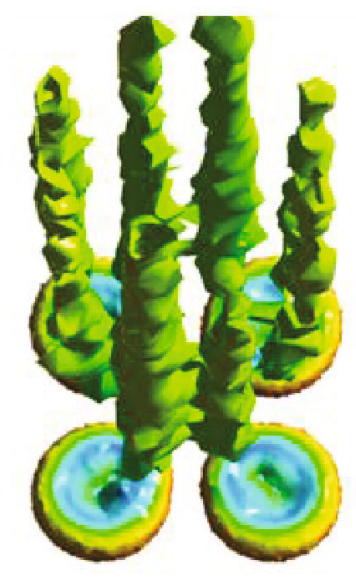

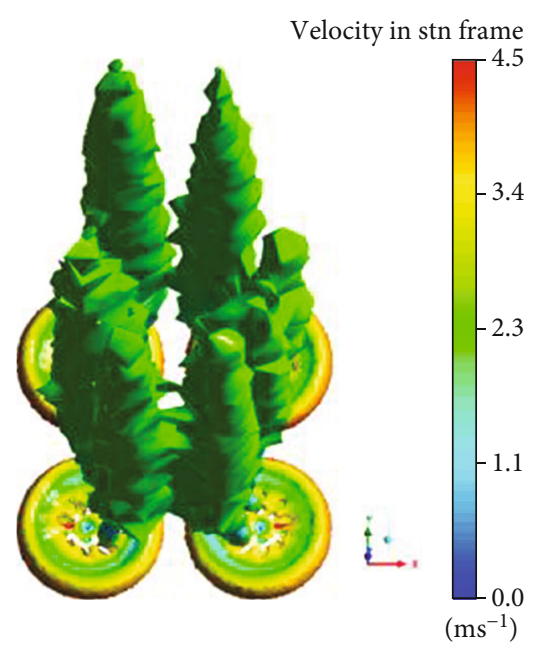

(d)

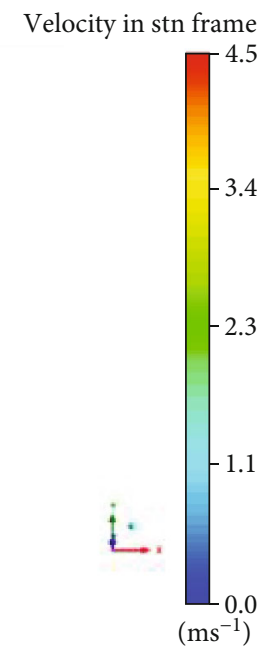

(e)

FIGURE 19: Vorticity distribution: (a) without wind disturbance; (b) horizontal wind $-2.5 \mathrm{~m} / \mathrm{s}$; (c) horizontal wind-4.0 m/s; (d) vertical wind $-2.5 \mathrm{~m} / \mathrm{s}$; (e) vertical wind $-4.0 \mathrm{~m} / \mathrm{s}$.

for the quadrotor. However, this situation is opposite with the influence of vertical wind, resulting in reduced aerodynamic performance for quadrotor.

To further analyze the reason why the thrust increment of quadrotor is different with horizontal wind disturbance, especially for rotor speed at $1700 \mathrm{RPM}$, the pressure contour of blade tip is shown in Figure 18.

From Figure 18, it is clear that the area of the negative pressure is obviously larger at 1700 RPM compared with other rotor speeds, which brings a larger thrust for 
quadrotor. However, the positive pressure increases significantly at a higher rotor speed of 2100 RPM because of strong aerodynamic interference, resulting in a smaller pressure difference, which reduces the thrust of quadrotor. This validates the experimental results where the thrust of quadrotor obviously improved at 1700 RPM with horizontal wind disturbance.

The vorticity distribution of the quadrotor with wind disturbance at $s=1.8$ is shown in Figure 19 .

As shown in Figure 19(a), without the effects of wind gust, the vorticity of rotors is independent with each other, and the shape of the vorticity is uniform and regular without interference from each other. As shown in Figures 19(b) and 19 (c), it can be seen that the shape of the original vortices is destroyed when the horizontal wind is introduced. The wakes and downwash flows of the front rotors posed strong interference on the downstream rotors. As the horizontal wind speed increases, the aerodynamic interference is more intense. As shown in Figures 19(d) and 19(e), with the influence of the vertical wind, the vortices formed above the rotors, where the vortices began to move and deform. Combined with the experimental results, it can be speculated that this will adversely affect the aerodynamic performance of the quadrotors, resulting in the reduction of thrust and power loading of rotors.

\section{Conclusions}

In order to study the wind effect on aerodynamic performance of quadrotor MAV with different rotor arrangement, both wind tunnel tests and numerical simulations are performed in this paper. Conclusions are as follows:

(1) More thrust is obtained with proper rotor interference compared with the four isolated rotors. With smaller rotor spacing, the interaction between the downwash flows and the mutual induction between the wakes are very intense, resulting in loss in thrust and increase in power consumption. Also, with an increasing spacing ratio, the quadrotor aircraft has the best aerodynamic performance with larger thrust and smaller power consumption especially for $s=1.8$

(2) Compared with no wind gust, the thrust of quadrotor increases obviously with the influence of horizontal wind. Especially for the horizontal wind at $2.5 \mathrm{~m} / \mathrm{s}$, the thrust increased $12 \%$ with a power reduction of $2.6 \%$ which indicated that the quadrotor obtained a good wind resistance at $2.5 \mathrm{~m} / \mathrm{s}$ in horizontal wind. However, when the vertical wind is introduced, the thrust of the quadrotors is severely weakened with thrust reduction around $10 \%$ which also indicated that the quadrotor may need control compensation in vertical wind with a decreased performance

(3) Compared with vertical wind disturbance, the vortices formed at the blade tip are more likely to be dispersed by horizontal wind disturbance, which may bring better aerodynamic performance for quadrotor. With the influence of vertical wind, the original vortices without wind disturbance move upwards and deform, which causes the streamline to change from circumferential flow to axial flow. This may have a negative effect on the aerodynamic performance of the quadrotor, resulting in the reduction of thrust and the increment of power consumption

Future investigations will attempt to study the quadrotor with an antidisturbance remote sensing platform which has been proved with high ground resolution, rapid response with simulation, and field testing.

\section{Data Availability}

The data used to support the findings of this study are included within the article.

\section{Conflicts of Interest}

The authors declare no conflict of interest.

\section{Authors' Contributions}

YL carried out the experiments and analyzed the experimental results; YH did the simulations; HW wrote the manuscript with the assistance of YL.

\section{Acknowledgments}

This research was supported by the National Natural Science Foundation of China (Grant No. 51505087), Fuzhou University Jinjiang Science and Education Park (No. 2019-JJFDKY-59), and Fujian Provincial Industrial Robot Basic Components Technology Research and Development Center (2014H2004). The authors thank the Key Laboratory of Fluid Power and Intelligent Electro-Hydraulic Control (Fuzhou University), Fujian Province University, and Fuzhou University Jinjiang Science and Education Park for applying the experimental field.

\section{References}

[1] P. Pounds, R. Mahony, and P. Corke, "Modelling and control of a large quadrotor robot," Control Engineering Practice, vol. 18, no. 7, pp. 691-699, 2010.

[2] U. Ansari, A. H. Bajodah, and B. Kada, "Development and experimental investigation of a quadrotor's robust generalized dynamic inversion control system," Nonlinear Dynamics, vol. 96, no. 2, pp. 1541-1557, 2019.

[3] H. Teng, I. Ahmad, and M. S. M. Alamgir, "3D optimal surveillance trajectory planning for multiple UAVs by using particle swarm optimization with surveillance area priority," IEEE Access, vol. 18, pp. 86316-86327, 2020.

[4] M. Atmaca, B. Cetin, and E. Yilmaz, "CFD analysis of unmanned aerial vehicles (UAV) moving in flocks," Acta Physica Polonica A, vol. 135, no. 4, pp. 694-696, 2019.

[5] M. Thompson, S. Watkins, C. White, and J. Holmes, "Spanwise wind fluctuations in open terrain as applicable to small flying craft," Aeronautical Journal, vol. 115, no. 1173, pp. $693-701,2011$. 
[6] A. Lidor, D. Weihs, and E. Sher, "Novel propulsion systems for micro aerial vehicles," Journal of Propulsion and Power, vol. 35, no. 1, pp. 243-267, 2019.

[7] L. N. N. T. Ha, D. H. P. Bui, and S. K. Hong, "Nonlinear control for autonomous trajectory tracking while considering collision avoidance of UAVs based on geometric relations," Energies, vol. 12, no. 8, p. 1551, 2019.

[8] H. Liu, T. Ma, F. L. Lewis, and Y. Wan, "Robust formation control for multiple quadrotors with nonlinearities and disturbances," IEEE transactions on cybernetics, vol. 50, no. 4, pp. 1362-1371, 2020.

[9] Q. Z. Xu, Z. S. Wang, and Y. Li, "Fuzzy adaptive nonlinear information fusion model predictive attitude control of unmanned rotorcrafts," Aerospace Science and Technology, vol. 98, p. 105686, 2020.

[10] G. V. Raffo, M. G. Ortega, and F. R. Rubio, “An integral predictive/nonlinear Ho control structure for a quadrotor helicopter," Automatica, vol. 46, no. 1, pp. 29-39, 2010.

[11] S. Z. Sheng and C. W. Sun, "Control and optimization of a variable-pitch Quadrotor with minimum power consumption," Energies, vol. 9, no. 4, p. 232, 2016.

[12] A. Lidor, E. Sher, and D. Weihs, "Phase-change-materials as energy source for micro aerial vehicles (MAV)," Applied Thermal Engineering, vol. 65, no. 1-2, pp. 185-193, 2014.

[13] M. I. Woods, J. F. Henderson, and G. D. Lock, "Energy requirements for the flight of micro air vehicles," Aeronautical Journal, vol. 105, no. 1045, pp. 135-149, 2001.

[14] W. Craig, D. Yeo, and D. A. Paley, "Dynamics of a rotorpendulum with a small, stiff propeller in wind," in $A S M E$ 2016 Dynamic Systems and Control Conference, pp. 1-10, Minneapolis, MN, 2016.

[15] A. Mohamed, R. Carrese, D. F. Fletcher, and S. Watkins, "Scale-resolving simulation to predict the updraught regions over buildings for MAV orographic lift soaring," Journal of Wind Engineering and Industrial Aerodynamics, vol. 140, pp. 34-48, 2015.

[16] Y. Lei and J. L. Wang, "Aerodynamic performance of quadrotor UAV with non-planar rotors," Applied Sciences, vol. 9, no. 14 , p. $2779,2019$.

[17] Y. Lei and H. D. Wang, "Aerodynamic optimization of a micro quadrotor aircraft with different rotor spacings in hover," Applied Sciences, vol. 10, no. 4, p. 1272, 2020.

[18] J. Y. Hwang, M. K. Jung, and O. J. Kwon, "Numerical study of aerodynamic performance of a multirotor unmanned-aerialvehicle configuration," Journal of Aircraft, vol. 52, no. 3, pp. 839-846, 2015.

[19] D. H. Nguyen, Y. Liu, and K. Mori, "Experimental study for aerodynamic performance of quadrotor helicopter," Transactions of the Japan Society for Aeronautical and Space Sciences, vol. 61, no. 1, pp. 29-39, 2018.

[20] Y. Y. Guo, B. Jiang, and Y. M. Zhang, "A novel robust attitude control for quadrotor aircraft subject to actuator faults and wind gusts," IEEE/CAA Journal of Automatica sinica, vol. 5, no. 1, pp. 292-300, 2018.

[21] Y. Lei and R. Z. Lin, "Effect of wind disturbance on the aerodynamic performance of coaxial rotors during hovering," Measurement and Control, vol. 52, no. 5-6, pp. 665-674, 2019. 